\title{
A disrupted bulgeless satellite galaxy as counterpart of the ultraluminous X-ray source ESO 243-49 HLX-1
}

\author{
M. Mapelli ${ }^{1}$, F. Annibali ${ }^{2}$, L. Zampieri ${ }^{1}$, and R. Soria ${ }^{3}$ \\ 1 INAF - Osservatorio Astronomico di Padova, Vicolo dell'Osservatorio 5, 35122 Padova, Italy \\ e-mail: michela.mapelli@oapd.inaf.it \\ 2 INAF - Osservatorio Astronomico di Bologna, via Ranzani 1, 40127 Bologna, Italy \\ 3 International Centre for Radio Astronomy Research, Curtin University, GPO Box U1987, Perth, WA 6845, Australia
}

Received 2 August 2013 / Accepted 30 September 2013

\begin{abstract}
The point-like X-ray source HLX-1, close to the S0 galaxy ESO 243-49, is one the strongest intermediate-mass black hole candidates, but the nature of its counterpart is still puzzling. By means of $N$-body/smoothed particle hydrodynamics simulations, we investigate the hypothesis that the HLX-1 counterpart is the nucleus of a bulgeless satellite galaxy, which undergoes a minor merger with the S0 galaxy. We derived synthetic surface brightness profiles for the simulated counterpart of HLX-1 in six Hubble Space Telescope (HST) filters, ranging from far ultraviolet (FUV) to infrared wavelengths, and we compared them with the observed profiles. Our model matches the emission associated with the HLX-1 counterpart in all considered filters, including the bluer ones, even without requiring the contribution of an irradiated disc. The simulation can also account for an extended FUV emission, which is hinted at by the analysis of the F140LP HST filter. This matching is impossible to achieve by assuming either a bulgy satellite, a young star cluster, or an irradiated disc component.
\end{abstract}

Key words. galaxies: interactions - methods: numerical - galaxies: individual: ESO 243-49 - X-rays: individuals: HLX-1

\section{Introduction}

The X-ray source 2XMM J011028.1-460421 (hereafter HLX-1, Farrell et al. 2009) is one of the strongest intermediatemass black hole (IMBH) candidates. The peak X-ray luminosity $\left(\sim 10^{42} \mathrm{erg} \mathrm{s}^{-1}\right)$, combined with a disc-black body spectrum peaking at $T_{\text {in }} \sim 0.2 \mathrm{keV}$, indicates a black hole $(\mathrm{BH})$ mass $\sim 10^{4} M_{\odot}$ (Farrell et al. 2009; Davis et al. 2011; Servillat et al. 2011; Godet et al. 2012). In particular, the X-ray light curve shows a periodic "fast-rise exponential decay" (FRED) behaviour, with a semi-regular periodicity of $\sim 370$ days. This has been interpreted as the orbital period of the companion star (Lasota et al. 2011; Soria 2013). The recently observed transient radio emission further supports the IMBH hypothesis (Webb et al. 2010). HLX-1 is projected in the sky at $\sim 0.8 \mathrm{kpc}$ out of the plane and $\sim 3.3 \mathrm{kpc}$ away from the nucleus of the S0/a galaxy ESO $243-49$ (luminosity distance $\sim 96 \mathrm{Mpc}$ ). The galaxy ESO 243-49 is a member of the cluster Abell 2877 (e.g. Malumuth et al. 1992). The vicinity of HLX-1 with ESO 243-49 is confirmed by the redshift of the observed $\mathrm{H} \alpha$ emission line of the counterpart (Wiersema et al. 2010), although the velocity offset between this and the bulge of ESO 243-49 is $\approx 400 \mathrm{~km} \mathrm{~s}^{-1}$, close to the escape velocity from the S0 galaxy (Soria et al. 2013).

The optical counterpart of HLX-1 has been detected in various bands, from near infrared to far ultraviolet (FUV, Wiersema et al. 2010; Soria et al. 2010, 2012, hereafter S10, S12, respectively; Farrell et al. 2012, hereafter F12), but its nature remains puzzling. In particular, it is still unclear which fraction of the optical/ultraviolet (UV) emission comes from the X-rayirradiated outer accretion disc and which fraction from a compact stellar population surrounding the BH $(\mathrm{F} 12, \mathrm{~S} 12)$. If the contribution from the irradiated disc (ID) is negligible, (at least) a fraction of the stellar population surrounding the $\mathrm{BH}$ must be young ( 10 Myr), because the optical colours are distinctly blue. In contrast, if the optical counterpart is dominated by the ID, the stellar population must be old, so as not to overproduce the blue/UV emission.

Three main scenarios have been proposed to explain the nature of the counterpart: (i) the counterpart is a (young or old) star cluster (SC, e.g. S12; F12); (ii) the counterpart is the nucleus of a disrupted satellite galaxy, which is undergoing minor merger with the S0 galaxy (e.g., Bellovary et al. 2010; S10; Webb et al. 2010; Mapelli et al. 2012, hereafter M12; Mapelli et al. 2013, hereafter M13); or (iii) the counterpart is almost entirely due to the ID, and the IMBH is naked, apart from the donor star and perhaps a few other stars in its sphere of influence (S12; Zampieri et al., in prep.).

The contribution of the ID is predicted to be negligible only in the young SC scenario, while it has been found to be crucial in most other proposed scenarios (naked BH, old SC, and disrupted bulgy satellite galaxy). M13 showed that even the merger with a bulgy satellite galaxy requires a significant contribution from the ID, since many pericentre passages are needed to disrupt a stellar bulge and to make it consistent with the photometry of the HLX-1 counterpart in the infrared. On the other hand, after many pericentre passages, the satellite has lost all its gas, and there is no recent SF to contribute to the blue and UV filters.

In this paper, we focus on the minor merger scenario. While in M12 and M13 we assumed that the satellite galaxy has a bulge, in the current paper we simulate a bulgeless gas-rich satellite galaxy. This difference has important consequences, because pure disc satellite galaxies are disrupted faster than discs with bulges (Gnedin et al. 1999). The paper is organised as follows. 
In Sect. 2, we summarise the main scenarios proposed to explain the nature of HLX-1. In Sect. 3, the simulation methods are described. In Sect. 4, we discuss the main results. Our conclusions are presented in Sect. 5.

\section{The puzzling nature of the HLX-1 counterpart}

Henceforth, we assume that the IMBH in HLX-1 is surrounded by a substantial stellar population (investigating the case of a naked $\mathrm{BH}$ is beyond the aim of this paper, and will be considered in a follow-up study). We compare the two possible scenarios of a massive SC or a disrupted satellite galaxy, and discuss which one is more consistent with the observed optical photometry and at the same time more plausible from an evolutionary point of view.

Either a young $(\approx 10 \mathrm{Myr})$ or an old $(\approx 10-12 \mathrm{Gyr}) \mathrm{SC}$ is consistent with the available photometry (F12; S12; M13), depending on the contribution of the ID. In the young SC scenario, the presence of an IMBH may be explained with the runaway collapse of massive stars (e.g. Portegies Zwart \& MacMillan 2002), but there are two serious drawbacks: (i) it is difficult to explain the presence of a young SC far from the disc and in an early type galaxy (young SCs are a disc population and are more frequent in late-type galaxies, e.g. Portegies Zwart et al. 2010); (ii) if the optical variability of the counterpart (claimed by S12) is confirmed, then a large portion of the infrared to UV radiation comes from the ID, and the young SC cannot be more massive than $\approx 10^{4} M_{\odot}$. It is very difficult to explain the presence of a $\approx 10^{4} M_{\odot}$ IMBH in a $\approx 10^{4} M_{\odot}$ SC. Even if the optical variability is negligible, the mass of a 10-Myr young SC is unlikely to be $>10^{5} M_{\odot}$ for a realistic value of the extinction $\left(A_{\mathrm{V}} \approx 0.18\right)^{1}$.

In the old SC scenario, an IMBH might have formed through repeated mergers of stellar-mass BHs (Miller \& Hamilton 2002). The position of the HLX-1 counterpart is consistent with the distribution of globular clusters (which are a halo population). On the other hand, in this case a high (although not unrealistically high) level of disc emission is needed to explain the luminosity in the blue and UV bands.

Two other problems of the (either young or old) SC scenario are that (i) it is difficult to explain a $\sim 400 \mathrm{~km} \mathrm{~s}^{-1}$ relative velocity between the counterpart and the bulge of ESO 243-49; and (ii) we can hardly explain the formation of $\mathrm{a} \approx 10^{4} M_{\odot} \mathrm{IMBH}$ even in a $\sim 10^{6} M_{\odot}$ young or old SC (i.e. the maximum mass allowed for the counterpart by observations, F12, S12), since this mass ratio requires a very efficient formation pathway (e.g. the discussion in Portegies Zwart \& MacMillan 2002).

The minor merger scenario removes the latter difficulty, as the IMBH would belong to the low-mass tail of the distribution of super massive BHs (SMBHs), located at the centre of galaxies. There is increasing evidence of (both bulgy and bulgeless) galaxies hosting SMBHs with mass $\lesssim 10^{5} M_{\odot}$ at their centre (e.g. Filippenko \& Sargent 1989; Filippenko \& Ho 2003; Barth et al. 2004, 2009; Greene \& Ho 2004, 2007a,b; Satyapal et al. 2007, 2008, 2009; Dewangen et al. 2008; Shields et al. 2008; Desroches \& Ho 2009; Gliozzi et al. 2009; Jiang et al. 2011a,b; Secrest et al. 2012; Bianchi et al. 2013).

Furthermore, both the projected position and the high relative velocity of the HLX-1 counterpart are consistent with an ongoing merger. In addition, a recent minor merger is consistent

F12 estimate a young SC mass $4 \times 10^{6} M_{\odot}$ for $A_{\mathrm{V}} \approx 1.3$, but this extinction is rather extreme when compared with the hydrogen column density $\left(\approx\right.$ a few $10^{20}$ atoms $\left.\mathrm{cm}^{-2}\right)$ derived from disc-black-body fits to the X-ray spectra, and with the line-of-sight extinction $A_{\mathrm{V}}=0.053$. with the presence of prominent dust lanes around the nucleus of the S0 galaxy (e.g. Finkelman et al. 2010; Shabala et al. 2012) and the evidence of UV emission centred on its bulge (S10), indicating ongoing star formation (SF, e.g. Kaviraj et al. 2009). On the other hand, there is no smoking gun to indicate that ESO 243-49 underwent a merger (see the discussion in M13).

In M12 and M13, we simulated the merger between a bulgy gas-rich satellite galaxy and an S0 galaxy. We found that the simulations are in fair agreement with the integrated magnitude of the HLX-1 counterpart in the different bands only if (i) the merger is in a very late state $(>2.5$ Gyr since the first pericentre passage); and (ii) we assume that the ID emission is much stronger than the stellar component in all the filters but the near infrared one. These two requirements rise from the fact that many pericentre passages are needed to disrupt an old stellar bulge and to make it consistent with the photometry of the HLX-1 counterpart in the infrared. On the other hand, after many pericentre passages, the satellite has lost all its gas, and there is no recent SF to contribute to the blue and UV filters.

The main problem of the bulgy satellite scenario is that it cannot account for the entire emission from the counterpart: an ID component is needed to explain the blue, near UV (NUV), and FUV emission. Furthermore, the merger with a bulgy satellite cannot explain the extended FUV emission, which was recently claimed in M13.

M13 suggested that the FUV emission associated with the HLX-1 counterpart is more extended than implied by the point spread function (PSF), in the Hubble Space Telescope (HST) data. The current data do not allow us to exclude the possibility that the extended emission is connected with the background galaxy at $z=0.03$ (Wiersema et al. 2010), or even with ESO 243-49. In fact, the FUV emission of the counterpart of HLX-1 is consistent with that of a point-like source superimposed on an extended FUV "halo" (see Fig. 5 of M13). If the extended FUV emission is physically connected with the HLX-1 counterpart, the SC scenario and even the merger with a bulgy satellite galaxy are ruled out.

In the current paper, we investigate the scenario of a bulgeless satellite by means of $N$-body/smoothed particle hydrodynamics (SPH) simulations. Pure disc satellite galaxies are disrupted faster than disc galaxies with bulges, owing to their lower central density, which results in a smaller tidal radius (Gnedin et al. 1999; Feldmann et al. 2008). Thus, we want to check whether the merger with a bulgeless satellite galaxy can explain both the integrated magnitude in the bluer filters and the extended FUV emission.

\section{3. $\mathbf{N}$-body simulations}

As in M12 and M13, the initial conditions for both the primary galaxy and the secondary galaxy in the $N$-body model are generated by using an upgraded version of the code described in Widrow et al. (2008; see also Kuijken \& Dubinski 1995; Widrow \& Dubinski 2005). The code generates self-consistent disc-bulge-halo galaxy models, which are very close to equilibrium. In particular, the dark matter (DM) halo is modelled with a Navarro et al. (1996, NFW) profile. We use an exponential disc model (Hernquist 1993), while the bulge is spherical and comes from a generalisation of the Sérsic law (Widrow et al. 2008). The primary galaxy has a stellar spherical bulge and a stellar exponential disc, and has no gas. The satellite has no bulge, while it has a stellar exponential disc and a gaseous exponential disc. The total mass of the secondary is $\lesssim 1 / 20$ of the mass of the primary, classifying the outcome of the interaction as a minor merger. 
Table 1. Initial conditions of the $N$-body simulations.

\begin{tabular}{lcc}
\hline \hline Model galaxy properties $^{a}$ & Primary & Secondary \\
\hline$M_{\mathrm{DM}}\left(10^{11} M_{\odot}\right)$ & 7.0 & 0.3 \\
$M_{*}\left(10^{10} M_{\odot}\right)$ & 7.0 & 0.15 \\
$f_{\mathrm{b} / \mathrm{d}}\left(10^{8} M_{\odot}\right)$ & 0.25 & 0 \\
$M_{\text {gas }}$ & 0 & 1.38 \\
NFW scale radius $(\mathrm{kpc})$ & 6.0 & 3.0 \\
Disc scale length $(\mathrm{kpc})$ & 3.7 & 3.0 \\
Disc scale height $(\mathrm{kpc})$ & 0.37 & 0.30 \\
Bulge scale length $(\mathrm{kpc})$ & 0.6 & - \\
\hline
\end{tabular}

Notes. ${ }^{(a)} M_{\mathrm{DM}}$ and $M_{*}$ are the total DM mass and the total stellar mass of the galaxy, respectively. $f_{\mathrm{b} / \mathrm{d}}$ is the bulge-to-disc mass ratio. $M_{\text {gas }}$ is the total gas mass.

The masses and the scale lengths of the various components of the simulated galaxies are listed in Table 1.

The main orbital properties of the interaction are impact parameter $b=10.2 \mathrm{kpc}$, initial relative velocity between the centres-of-mass (CMs) of the two galaxies $v_{\text {rel }}=50 \mathrm{~km} \mathrm{~s}^{-1}$, orientation angles $\theta=-\pi / 2, \phi=0, \psi=2.94 \mathrm{rad}$ (for a definition of the angles, see Hut \& Bahcall 1983 and M12), specific orbital energy $E_{\mathrm{s}}=-2.03 \times 10^{4} \mathrm{~km}^{2} \mathrm{~s}^{-2}$, specific orbital angular momentum $L_{\mathrm{s}}=0.5 \times 10^{3} \mathrm{~km} \mathrm{~s}^{-1} \mathrm{kpc}$, and eccentricity $e=0.999$. The orbit is prograde; i.e., the orbital angular momentum of the satellite is aligned with the spin of the primary galaxy. The adopted orbits are nearly parabolic, in agreement with predictions from cosmological simulations (Tormen 1997; Wang et al. 2005; Zentner et al. 2005; Khochfar \& Burkert 2006; Wetzel 2011). Furthermore, this assumption is consistent with the measured velocity offset between the counterpart of HLX-1 and the bulge of ESO 243-49, which is close to the escape velocity from the $\mathrm{S} 0$ galaxy.

The particle mass in the primary galaxy is $10^{5} M_{\odot}$ and $10^{4} M_{\odot}$ for DM and stars, respectively. The particle mass in the secondary galaxy is $10^{4} M_{\odot}$ for DM and $7 \times 10^{2} M_{\odot}$ for both stars and gas. The total number of particles in the simulation is 18.7 Million. The softening length is $\epsilon=10 \mathrm{pc}$. We also ran a test simulation with softening length $\epsilon=30 \mathrm{pc}$, to make sure that spurious scatterings between particles of different mass are negligible, and we found no spurious effects (see e.g. Bate \& Burkert 1997).

As in M12 and M13, we simulate the evolution of the models with the $N$-body/SPH tree code GASOLINE (Wadsley et al. 2004). Radiative cooling, SF, and supernova blastwave feedback are enabled, as described in Stinson et al. (2009). In particular, SF occurs when cold $\left(<3 \times 10^{4} \mathrm{~K}\right)$, virialised gas reaches a threshold density $n_{\mathrm{SF}}=5$ atoms $\mathrm{cm}^{-3}$, and is part of a converging flow. SF proceeds at a rate

$$
\frac{\mathrm{d} \rho_{*}}{\mathrm{~d} t}=\epsilon_{\mathrm{SF}} \frac{\rho_{\mathrm{gas}}}{t_{\mathrm{dyn}}} \propto \rho_{\mathrm{gas}}^{1.5},
$$

(i.e. locally enforcing a Schmidt law), where $\rho_{*}$ and $\rho_{\text {gas }}$ are the stellar and gas densities, respectively, and $t_{\text {dyn }}$ is the local dynamical time. We choose $\epsilon_{\mathrm{SF}}=0.1$, in agreement with previous work (e.g. Mapelli \& Mayer 2012).

\section{Results}

\subsection{Gas morphology}

Figure 1 shows the projected distribution of the gas component of the simulated satellite galaxy at $t=100 \mathrm{Myr}$ after the first

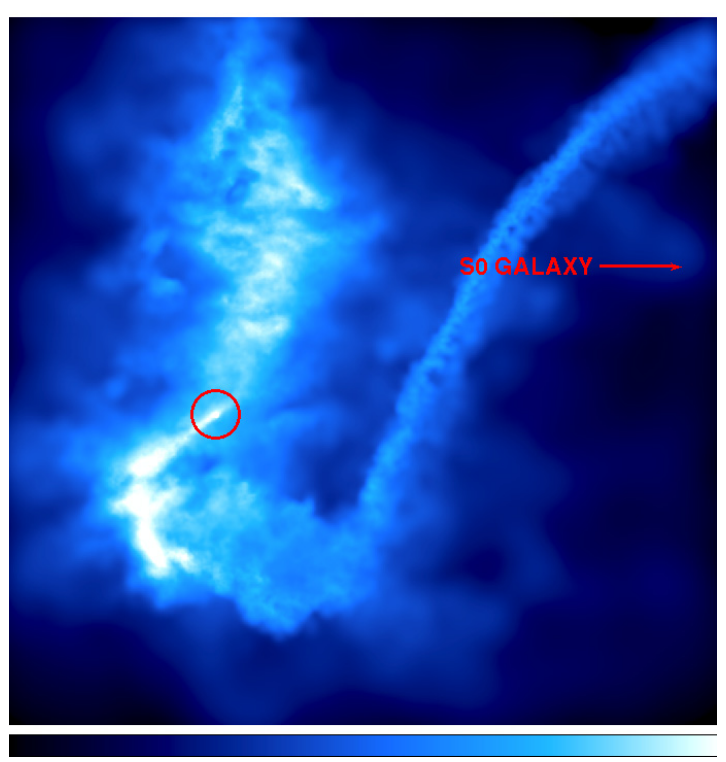

Fig. 1. Colour-coded map: projected mass density of gas in the simulated satellite galaxy at $t=100 \mathrm{Myr}$ after the first pericentre passage. The satellite is seen face-on. The scale of the colour-coded map is logarithmic, ranging from $2.2 \times 10^{-9} M_{\odot} \mathrm{pc}^{-3}$ (black) to $2.2 \times$ $10^{-2} M_{\odot} \mathrm{pc}^{-3}$ (white). The frame size is $60 \times 60 \mathrm{kpc}$. The red circle marks the position of the nucleus of the satellite galaxy. The direction of the $\mathrm{S} 0$ bulge is indicated by the red arrow.

pericentre passage. The satellite galaxy is shown face-on. The two tidal lobes are very evident, as is the tidal stream connecting the satellite with the bulge of the primary. The position of the nucleus of the satellite galaxy is marked. Figure 2 again shows the projected distribution of the gas component of the simulated satellite galaxy at $t=100 \mathrm{Myr}$, but now the satellite is seen edgeon and is rotated to match the observed position of the HLX-1 counterpart with respect to the SO galaxy.

Figures 1 and 2 indicate that the distribution of the atomic hydrogen close to ESO 243-49 should show some interesting features, if the counterpart of HLX-1 is the nucleus of a recently disrupted satellite. We notice that the projected distribution of the gas in Fig. 2 qualitatively recalls the distribution of FUV emission in Fig. 4 of M13, although the two figures cannot be directly compared. The main difference is that while the FUV emission has a peak in the bulge of ESO 243-49, there is no concentration of gas at the centre of the SO galaxy in our simulation. This happens because the gas stripped from the satellite galaxy did not have enough time to sink to the centre of the primary galaxy: M12 and M13 showed that it takes $\gtrsim 1$ Gyr (since the first pericentre passage) for the stripped gas to reach the nucleus of the S0 galaxy.

The absence of gas at the centre of the simulated S0 galaxy might indicate that the SF observed at the centre of ESO 243-49 is due to residual gas bound to the S0 galaxy, rather than to the gas stripped from the satellite (e.g. Temi et al. 2009; M13).

\subsection{The stellar component and the surface brightness profiles}

Part of the gas was converted into young stars by applying the SF recipes described in Sect. 3. We find that the simulated mass of young stars in the inner $0.4 \operatorname{arcsec}$ of the satellite is $M_{\mathrm{y}}\left(<0.4^{\prime \prime}\right)=$ $2.5 \times 10^{4} M_{\odot}$ at $t=100 \mathrm{Myr}$, corresponding to $\sim 6$ per cent of the total stellar mass within $0.4 \operatorname{arcsec}\left(=3.9 \times 10^{5} M_{\odot}\right)$. 


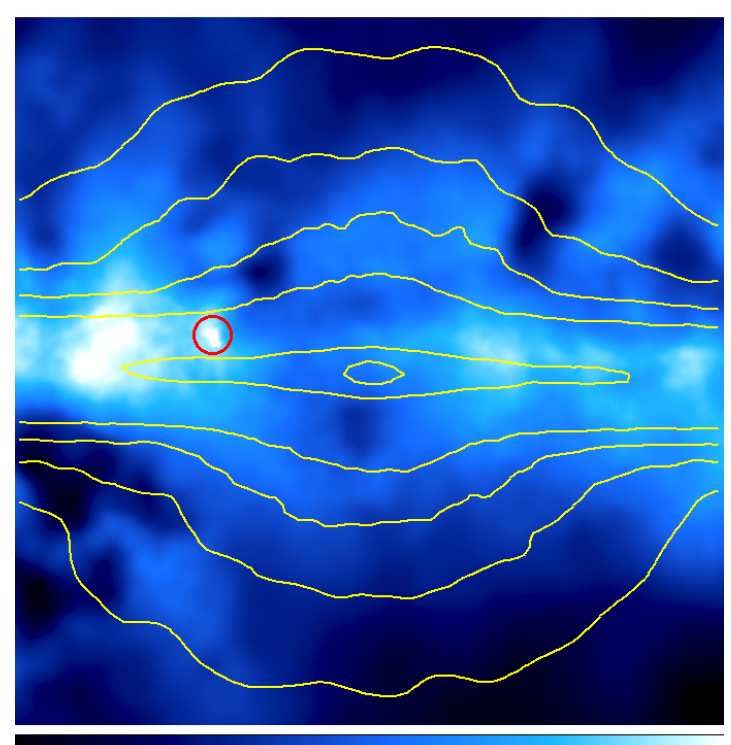

Fig. 2. Colour-coded map: projected mass density of gas in the simulated satellite galaxy at $t=100 \mathrm{Myr}$ after the first pericentre passage. Both the satellite and the S0 galaxy are seen edge-on. The scale of the colour-coded map is logarithmic, ranging from $2.2 \times$ $10^{-9} M_{\odot} \mathrm{pc}^{-3}$ (black) to $7.0 \times 10^{-2} M_{\odot} \mathrm{pc}^{-3}$ (white). The frame size is $16 \times 16 \mathrm{kpc}$. The red circle marks the position of the nucleus of the satellite galaxy. The yellow contours show the projected density of stars in the So galaxy.

We then derive synthetic fluxes (in the six HST filters) by using the single stellar population (SSP) models based on the tracks of Marigo et al. (2008), with the Girardi et al. (2010) case A correction for low-mass, low-metallicity asymptotic giant branch stars ${ }^{2}$. For simplicity, we assume that all the stars already present in the initial conditions of the simulation have an age $t_{\text {old }}=12 \mathrm{Gyr}$ and that all the young stars that formed as a consequence of the merger have an age $t_{\mathrm{y}}=10 \mathrm{Myr}$.

The results are shown in Fig. 3 and in Table 2. In Fig. 3, we compare the synthetic surface brightness profiles with the observed surface brightness profiles of the HLX-1 counterpart (derived in M13). We recall that one of the major issues about the optical counterpart of HLX-1 (as highlighted in F12, S12, $\mathrm{M} 12$, and M13) is the subtraction of the light coming from the ESO 243-49. The contamination from ESO 243-49 is particularly strong in the infrared (F160W), where it is impossible to recover a surface brightness profile for the HLX-1 counterpart, and it is still dominant in the F775W and in the F555W filters, while it is less critical in the bluer filters. In M13, we used and compared two approaches to removing the background. In the first approach, we computed the background in an annulus ${ }^{3}$ of width 0.08 arcsec at $r>1$ arcsec. In the second approach, we created a Gaussian smoothed image of the S0 galaxy and then subtracted it to the original frame. The first approach is more conservative and does not smear out possible faint irregular features. On the other hand, the first approach might fail to remove the contribution of the S0 galaxy at large radii $(r>0.2$ arc$\mathrm{sec}$ ). The first approach works fine for the bluer filters (F140LP, F300X, and F390W), but it overestimates the integrated light of

\footnotetext{
2 http://stev.oapd.inaf.it/cgi-bin/cmd_2.3

3 To account for the uncertainties due to the highly variable S0 background, we repeated the computation by varying the position of the background annulus between 1.0 and 1.4 arcsec in steps of $0.2 \operatorname{arcsec}$, and then averaging the results (see M13).
}

the HLX-1 counterpart in the redder ones (F555W and F775W) with respect to previous work (F12 and references therein). Thus, even if the level of contamination from ESO 243-49 is still a matter of debate, in M13 and in this paper we take the results of the second approach as reference values for the F555W and F775W filters.

Figure 3 shows our fiducial simulation (solid line) and our fiducial run convolved with the observed $\mathrm{PSF}^{4}$ (dotted line). We warn that the convolution may lead to underestimating the flux of the simulation in the inner bins, where the simulation is dominated by softening effects. Thus, we report both the nonconvolved (solid line) and the convolved (dotted line) profile to give an idea of the upper and lower cases for the inner bins. We notice that the effect of the PSF convolution is dramatic in the case of the F160W filter, where the PSF full width at half maximum is $\gtrsim 3$ times the value of the other filters.

Figure 3 shows the uncertainty of the simulation (yellow shaded area), which is due to the combined effects of stochastic fluctuations and spatial resolution. In particular, it accounts for the differences between the fiducial simulation and a test run, which differ only for the softening length $(\epsilon=10$ and $30 \mathrm{pc}$ in the fiducial simulation and in the test run, respectively, see Sect. 3). As we did not find any significant discrepancy in the energy distribution of particles between the fiducial and the test run, we can consider the differences between the two runs at $r>30 \mathrm{pc}$ as purely stochastic fluctuations, owing to the low number of particles in the nucleus of the satellite. Thus, the uncertainty of the simulation (represented by the shaded area) is larger (i) at $r<30 \mathrm{pc}$ because of the different softening length;

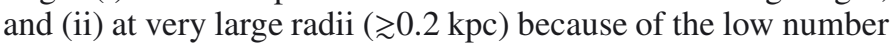
of particles, which increases the effect of stochastic fluctuations.

The simulation matches the observed profile of the HLX-1 counterpart in all the considered filters, even the bluer ones (F390W, F300X, and F140LP), without requiring any contribution from an ID component. The simulation also matches the extended profile of the HLX-1 counterpart in the FUV filter. As shown in M13, this result is impossible to achieve by assuming either a bulgy satellite or an ID component. Thus, the minor merger with a bulgeless gas-rich satellite is the only way to explain the extended FUV emission, if this is associated with the HLX-1 counterpart.

In Table 2, we report the integrated magnitude within 0.4 arcsec estimated from the fiducial simulation, and we compare it with the observational values derived in M13. There is a fair agreement between the simulation and the data in the FUV (F140LP) and in the infrared (F160W) filter, while there is a larger discrepancy in the other filters.

We stress that the synthetic surface brightness profiles shown in Fig. 3 and the integrated magnitudes reported in Table 2 are not attempts to fit the observational data, because we ran just one simulation and we have no free parameters to play with. Given the large uncertainty connected with stochastic fluctuations, we do not think that it is meaningful to run a wider grid of simulations, until more constraints about the orbit and the gas content of the HLX-1 counterpart become available.

Furthermore, we do not add any ID component, but it is likely that there is some contribution from the accretion disc. Adding an ID component to the stellar population component would increase the range of models that match the data, but would imply additional free parameters.

\footnotetext{
4 As in M13, we approximate the PSF as a two-dimensional Gaussian with the same full-width at half maximum as the observed PSF.
} 

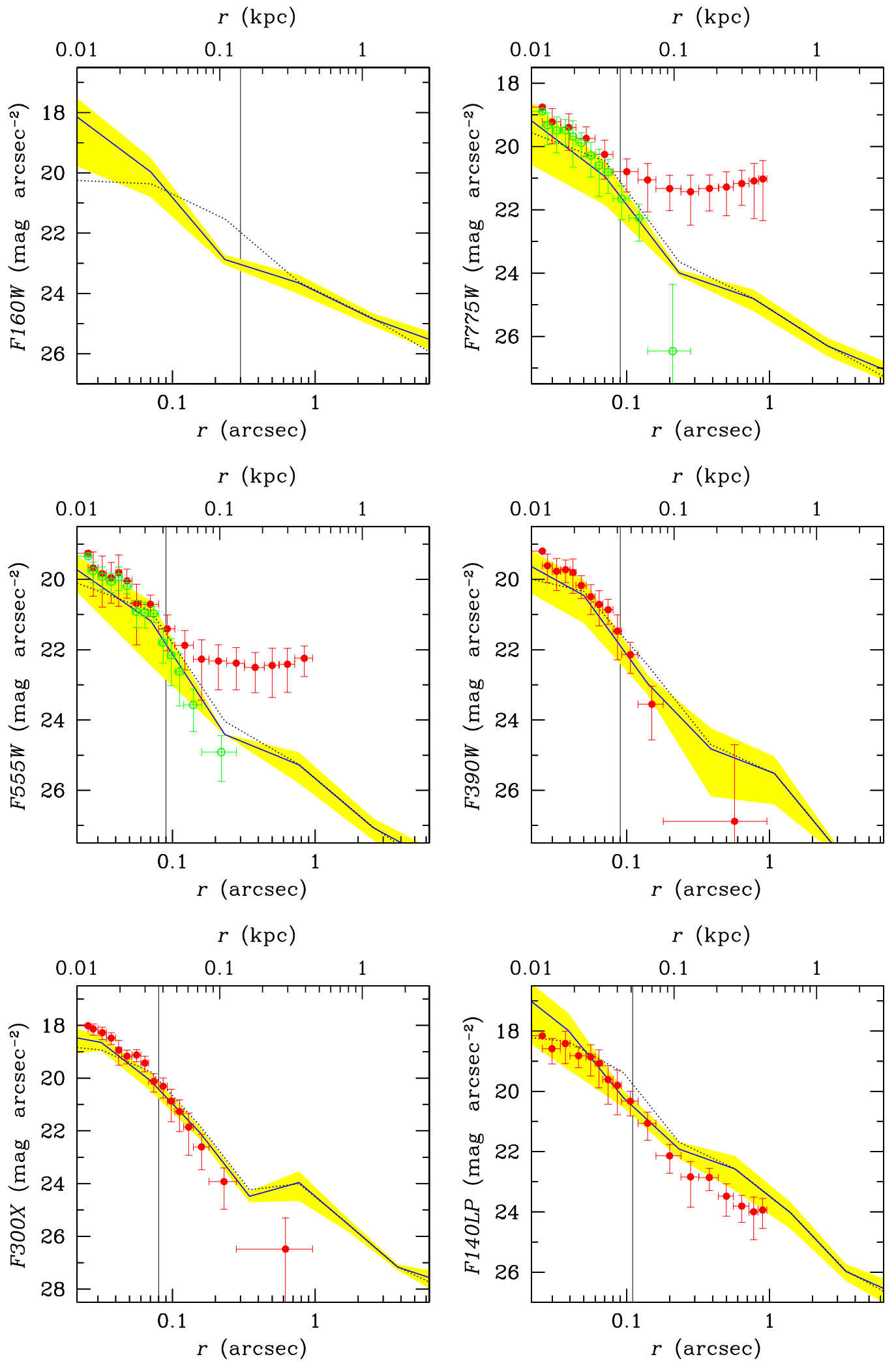

Fig. 3. Surface brightness profiles of the HLX-1 counterpart in the simulation (lines) and in the HST observations (circles). From left to right and from top to bottom: filter F160W, F775W, F555W, F390W, F300X, and F140LP. In all panels, solid blue line: simulated profile of the satellite galaxy at $t=100 \mathrm{Myr}$ after the first pericentre passage. We assume $A_{V}=0.18$ for the simulation. The dotted black line is the simulated profile convolved with the observed PSF. The yellow shaded area shows the uncertainty in the simulation due to stochastic fluctuations and to spatial resolution (see the text for details). The filled red circles were obtained in M13 by subtracting the background according to the first approach (as described in M13). In the F775W and F555W filters, the open green circles were obtained by subtracting the background according to the second approach, as described in M13. We do not show the observed profile in F160W as the background of the S0 galaxy is much stronger than the flux of the HLX-1 counterpart in this filter. The error bars of the data points are at $1 \sigma$. Vertical solid line: PSF full-width at half maximum, as derived in M13. 
Table 2. Simulated magnitudes within 0.4 arcsec, compared with the observed magnitude within $0.4 \operatorname{arcsec}, m\left(<0.4^{\prime \prime}\right)$.

\begin{tabular}{lccc}
\hline \hline Filter & $\begin{array}{c}\text { Model } \\
\left(A_{V}=0\right)\end{array}$ & $\begin{array}{c}\text { Model } \\
\left(A_{V}=0.18\right)\end{array}$ & $m\left(<0.4^{\prime \prime}\right)$ \\
\hline F140LP & 21.9 & 22.2 & $22.21 \pm 0.03$ \\
F300X & 23.0 & 23.3 & $22.80 \pm 0.05$ \\
F390W & 24.1 & 24.3 & $24.04 \pm 0.05$ \\
F555W & 24.4 & 24.6 & $24.11 \pm 0.05$ \\
F775W & 24.0 & 24.2 & $23.64 \pm 0.15$ \\
F160W & 23.2 & 23.3 & $23.49 \pm 0.26$ \\
\hline
\end{tabular}

Notes. All the reported magnitudes are in Vegamag. In Cols. 2 and 3 we show the magnitude of the fiducial simulation, assuming $A_{V}=0$ and $A_{V}=0.18$ (M13, S12), respectively. The uncertainty in the simulated magnitude is $\approx 0.5 \mathrm{mag}$. In Col. 4 we report the observed magnitude within 0.4 arcsec, from Table 1 of M13, to facilitate the comparison.

\section{Conclusions}

In this paper, we have investigated the hypothesis that the HLX-1 counterpart is the nucleus of a bulgeless satellite galaxy undergoing a minor merger with the S0 galaxy. A bulgeless satellite is disrupted faster (due to its lower central density, Gnedin et al. 1999) than a bulgy satellite. For this reason, the simulation presented in this paper is able to reproduce the photometry of the counterpart of HLX-1 in all the HST filters, including the bluer ones (F390W, F300X, and F140LP). Instead, a simulated bulgy satellite can only account for the emission in the redder filters (F160W and F775W, see M13), because the old stars dominate the stellar population. For a bulgy satellite to be consistent with the counterpart of HLX-1, we need to assume that most of the optical and UV emission comes from an ID (M13), while a bulgeless satellite scenario implies very mild or no contribution from the disc.

M13 have recently suggested that the FUV emission associated with the HLX-1 counterpart may be extended. This claim and the nature of the extended emission (i.e. whether it is physically connected with the HLX-1 counterpart or with the ESO 243-49 or with a background galaxy) are still being debated. Our simulations show that a bulgeless satellite galaxy can match the extended FUV emission surrounding the HLX-1 counterpart. This matching is impossible to achieve by assuming either a bulgy satellite (M13), a young SC, or an ID component. If the satellite has a bulge, it takes a long time to be disrupted ( $\gtrsim 2.5$ Gyr since the first pericentre passage), and the $\mathrm{SF}$ in the satellite is quenched by gas stripping, before the flux of the old stellar component becomes consistent with the observed one in the infrared.

We chose a realistic set of orbital parameters for the simulation presented in this paper, and we showed that the results match the photometry of the HLX-1 counterpart. We expect that other choices of the orbital parameters (eccentricity, angular momentum, specific energy) and of the properties of the satellite (especially stellar and gas mass) may produce results in agreement with the observations. While it is prohibitive to cover the entire parameter space with sufficiently high-resolution simulations, a number of different orbital parameters and satellite properties deserve to be investigated in a forthcoming study. This will be particularly helpful if more accurate photometric and spectral constraints on the HLX-1 counterpart become available.

We add no ID component in our model, but it is likely that the disc emission contributes to the observed flux. It is essential to establish whether the HLX-1 counterpart is variable, since this is the only clue to disentangle an ID from the stellar population component. Finally, our simulations show that tidal tails should be evident in the gas component (Figs. 1 and 2), if the HLX-1 counterpart is the nucleus of a bulgeless satellite galaxy. Thus, observations of the atomic hydrogen and of its kinematics can contribute to shedding light on the nature of the HLX-1 counterpart.

Acknowledgements. We thank the anonymous referee for their comments that improved the manuscript. We also thank the developers of GASOLINE (especially Wadsley, Quinn, and Stadel). We thank L. Widrow for providing the code for generating the initial conditions, Girardi and Bressan for providing the SSP models, and Ripamonti for useful discussions. To analyse simulation outputs, we made use of the software TIPSY ${ }^{5}$. We acknowledge the CINECA Award N. HP10CLI3BX and HP10B3BJEW, 2011 for the availability of high-performance computing resources and support. We thank Cormac Reynolds for his precious technical support and for allowing us to run on CUPPA, the Beowulf cluster of the Curtin Institute of Radio Astronomy (CIRA) at Curtin University. M.M. and L.Z. acknowledge financial support from the Italian Ministry of Education, University and Research (MIUR) through grant FIRB 2012 RBFR12PM1F ("New perspectives on the violent Universe: unveiling the physics of compact objects with joint observations of gravitational waves and electromagnetic radiation"), and from INAF through grant PRIN-2011-1 ("Challenging Ultraluminous X-ray sources: chasing their black holes and formation pathways").

\section{References}

Barth, A. J., Ho, L. C., Rutledge, R. E., \& Sargent, W. L. W. 2004, ApJ, 607, 90 Barth, A. J., Strigari, L. E., Bentz, M. C., Greene, J. E., \& Ho, L. C. 2009, ApJ, 690, 1031

Bate, M. R., \& Burkert, A. 1997, MNRAS, 288, 1060

Bellovary, J. M., Governato, F., Quinn, Th. R., et al. 2010, ApJ, 721, L148

Bianchi, S., Piconcelli, E., Pérez-Torres, M. Á., et al. 2013, MNRAS, 435, 2335

Davis, Sh. W., Narayan, R., Zhu, Y., et al. 2011, ApJ, 734, 111

Desroches, L.-B., \& Ho, L. C. 2009, ApJ, 690, 267

Dewangen, G. C., Mathur, S., Griffiths, R. E., \& Rao, A. R. 2008, ApJ, 689, 726

Farrell, S. A., Webb, N. A., Barret, D., Godet, O., \& Rodrigues, J. M. 2009, Nature, 460, 73

Farrell, S. A., Servillat, M., Pforr, J., et al. 2012, ApJ, 747, L13 (F12)

Feldmann, R., Mayer, L., \& Carollo, C. M. 2008, ApJ, 684, 1062

Filippenko, A. V., \& Ho, L. C. 2003, ApJ, 588, L13

Filippenko, A. V., \& Sargent, W. L. W. 1989, ApJ, 342, L11

Finkelman, I., Brosch, N., Funes, J. G., Kniazev, A. Y., \& Väisänen, P. 2010, MNRAS, 407, 2475

Girardi, L., Williams, B. F., Gilbert, K., et al. 2010, ApJ, 724, 1030

Gliozzi, M., Satyapal, S., Erancleous, M., Titarchuk, L., \& Cheung, C. C. 2009, ApJ, 700, 1759

Gnedin, O. Y., Hernquist, L., \& Ostriker, J. P. 1999, ApJ, 514, 109

Godet, O., Plazolles, B., Kawaguchi, T., et al. 2012, ApJ, 752, 34

Greene, J. E., \& Ho, L. C. 2004, ApJ, 610, 722

Greene, J. E., \& Ho, L. C. 2007a, ApJ, 667, 131

Greene, J. E., \& Ho, L. C. 2007b, ApJ, 670, 92

Hernquist, L. 1993, ApJS, 86, 389

Hut, P., \& Bahcall, J. N. 1983, ApJ, 268, 319

Jiang, Y., Greene, J. E., \& Ho, L. C. 2011a, ApJ, 737, L45

Jiang, Y., Greene, J. E., Ho, L. C., Xiao, T., \& Barth, A. J. 2011b, ApJ, 742, 68

Kaviraj, S., Peirani, S., Khochfar, S., Silk, J., \& Kay, S. 2009, MNRAS, 394, 1713

Khochfar, S., \& Burkert, A. 2006, A\&A, 445, 403

Kuijken, K., \& Dubinski, J. 1995, MNRAS, 277, 1341

Lasota, J.-P., Alexander, T., Dubus, G., et al. 2011, ApJ, 735, 89

Malumuth, E. M., Kriss, G. A., Dixon Van Dyke, W., Ferguson, H. C., \& Ritchie, Ch. 1992, ApJ, 104, 495

Mapelli, M., \& Mayer, L. 2012, MNRAS, 420, 1158

Mapelli, M., Zampieri, L., \& Mayer, L. 2012, MNRAS, 423, 1309 (M12)

Mapelli, M., Annibali, F., Zampieri, L., \& Soria, R. 2013, MNRAS, 433, 849 (M13)

Marigo, P., Girardi, L., Bressan, A., et al. 2008, A\&A, 482, 883

Miller, M. C., \& Hamilton, D. P. 2002, MNRAS, 330, 232

\footnotetext{
5 http://www-hpcc.astro.washington.edu/tools/tipsy/ tipsy.html
} 
M. Mapelli et al.: A bulgeless satellite as counterpart of HLX-1

Navarro, J. F., Frenk, C. S., \& White, S. D. M. 1996, ApJ, 462, 563 Portegies Zwart, S. F., \& McMillan, S. L. W. 2002, ApJ, 576, 899 Portegies Zwart, S. F., McMillan, S. L. W., \& Gieles, M. 2010, ARA\&A, 48, 431

Satyapal, S., Vega, D., Heckman, T., OHalloran, B., \& Dudik, R. 2007, ApJ, 663 L9

Satyapal, S., Vega, D., Dudik, R. P., Abel, N. P., \& Heckman, T. 2008, ApJ, 677, 926

Satyapal, S., Böker, T., McAlpine, W., et al. 2009, ApJ, 704, 439

Secrest, N. J., Satyapal, S., Gliozzi, M., et al. 2012, ApJ, 753, 38

Servillat, M., Farrell, S. A., Lin, D., et al. 2011, ApJ, 743, 6

Shabala, S. S., Ting, Y.-S., Kaviraj, S., et al. 2012, MNRAS, 423, 59

Shields, J. C., Walcher, J. C., Böker, T., \& Ho, L. C. 2008, ApJ, 682, 104

Soria, R. 2013, MNRAS, 428, 1944

Soria, R., Hau, G. K. T., Graham, A. W., et al. 2010, MNRAS, 405, 870 (S10)
Soria, R., Hakala, P., Hau, G., Gladstone, J., \& Kong, A. 2012, MNRAS, 420, 3599 (S12)

Soria, R., Hau, G. K. T., \& Pakull, M. W. 2013, ApJ, 768, L22

Stinson, G., Dalcanton, J. J., Quinn, T., et al. 2009, 395, 1455

Temi, P., Brighenti, F., \& Mathews, W. G. 2009, ApJ, 695, 1

Tormen, G. 1997, MNRAS, 290, 411

Wadsley, J. W., Stadel, J., \& Quinn, T. 2004, New Astron., 9, 137

Wang, H. Y., Jing, Y. P., Mao, S., \& Kang, X. 2005, MNRAS, 364, 424

Webb, N. A., Barret, D., Godet, O., et al. 2010, ApJ, 712, L107

Wetzel, A. R. 2011, MNRAS, 412, 49

Widrow, L. M., \& Dubinski, J. 2005, ApJ, 631, 838

Widrow, L. M., Pym, B., \& Dubinski, J. 2008, ApJ, 679, 1239

Wiersema, K., Farrell, S. A., Webb, N. A., et al. 2010, ApJ, 721, L102

Zentner, A. R., Berlind, A. A., Bullock, J. S., Kravtsov A. V., \& Wechsler R. H. 2005, ApJ, 624, 505 